\section{Evidence-Based Infection Control Planning Based on National Healthcare- Associated Infection Prevalence Data}

\author{
Jacqueline Reilly, PhD; Sally Stewart, MSc; \\ Gwen Allardice, PhD; Shona Cairns, BSc; \\ Lisa Ritchie, BA; Julie Bruce, PhD
}

This study identifies factors associated with a high prevalence of healthcare-associated infection (HAI) in the Scottish inpatient population, on the basis of the Scotland National HAI Prevalence Survey data set. The multivariate models developed can be used to predict HAI prevalence in specific patient groups to help with planning and policy in infection control.

Healthcare-associated infection (HAI) is a major public health problem and contributes to significant morbidity and mortality worldwide. Multiple factors contribute to the increasing risk of HAI, including an aging population, ${ }^{1,2}$ emerging and reemerging antimicrobial-resistant microorganisms, ${ }^{3}$ and widespread use of complex therapeutic interventions.

Efforts to address HAI should be targeted where there is the most potential for prevention and control. Surveillance data can provide the opportunity for an evidence-based approach to policy and planning in this regard. Many countries, including the United Kingdom, have established comprehensive surveillance programs to monitor infection rates and analyze trends over time. These are mostly incidence-based prospective studies that record data on specified organisms or infection types. ${ }^{4}$ However, incidence-based programs require regular review to ensure that surveillance is targeted, particularly in the light of the emergence of drug-resistant organisms, to ensure maximum impact, benefit, and costeffectiveness.

Prevalence surveys are a relatively rapid and cost-effective way to estimate the magnitude and burden of HAI. ${ }^{5}$ These surveys can provide data to inform priority setting at the national and hospital level. In 2005, the Scottish government commissioned the first national HAI prevalence survey of all acute care hospitals in Scotland. This survey revealed an infection prevalence of $9.5 \%$ (95\% confidence interval [CI], $8.8 \%-10.2 \%) .^{6}$ The aims of this study were to identify factors associated with HAI, on the basis of the Scotland National HAI Prevalence Survey dataset, and to develop a model to predict HAI prevalence in specific patient groups to help with planning and policy in infection control.

\section{METHODS}

The design and methodology of the Scotland National HAI Prevalence Survey have been described previously. ${ }^{6}$ In brief, this point prevalence survey included all 45 acute care hospitals in Scotland. The Centers for Disease Control and Prevention HAI case definitions were used, and the data were collected by independent trained data collectors. The prevalence of infection was defined as the percentage of the total number of patients surveyed who had infection.

Univariate and multivariate analyses were performed using the svy:logistic procedure of Stata (StataCorp), with "ward" as the primary sampling unit. The dependent variable was HAI status (yes or no). We investigated the effect on HAI prevalence of the following explanatory variables: patient age (50 years or less, $51-70$ years, $71-80$ years, or greater than 81 years), patient sex, hospital size (small [249 beds or fewer], medium [250-499 beds], or large [500 or more beds]), type of patient admission (planned or unplanned), hospital type (teaching, general, or obstetric), calendar quarter, and specialty of the consultant in charge of care. Predicted estimates of the prevalence of HAI were calculated using regression coefficients from the final model. ${ }^{7}$

\section{RESULTS}

The survey included a total of 11,608 inpatients, from 408 wards, from all 45 acute care hospitals in Scotland; 1,103 inpatients $(9.5 \%$; $95 \% \mathrm{CI}, 8.8 \%-10.2 \%)$ had a HAI on the day of survey. The study population was $58 \%$ female (median age, 74 years [interquartile range $\{\mathrm{IQR}\}, 56-83$ years]) and $42 \%$ male (median age, 69 years [IQR, 56-79 years]).

Univariate analyses found that infections were significantly more prevalent among men, among patients aged over 50 years, among patients receiving care from specialists in medical and elderly care, and among patients surveyed (ie, hospitalized) during winter months (November through January). Multivariate regression analysis revealed that male sex, age over 50 years, the receipt of care from specialists in surgical and elderly care, and participation in the survey (ie, hospitalization) during winter months were independently associated with high HAI prevalence (Table).

Examples of predicted HAI prevalence for 2 Scottish populations are given here by means of regression coefficient estimates. The predicted prevalence of HAI in a population of young (aged 50 years or less) female patients in an obstetrics unit during the summer is estimated to be $0.8 \%$. The predicted prevalence of HAI in an elderly male population (aged more than 81 years) in a unit for care of the elderly during the winter is $16.3 \%$.

\section{I S U S S ION}

Over the past few decades, characteristics of the hospitalized population have changed, with subsequent impact upon fac- 
тAвLE. Results of Multivariate Logistic Regression of Factors Associated With Healthcare-Associated Infection (HAI), According to the Scotland National HAI Prevalence Survey

\begin{tabular}{|c|c|c|c|c|c|}
\hline Variable & $\begin{array}{c}\text { No. of patients } \\
\text { surveyed }\end{array}$ & $\begin{array}{l}\text { No. of patients } \\
\text { with infection }\end{array}$ & Prevalence, $\%^{\mathrm{a}}$ & $\begin{array}{c}\text { Regression } \\
\text { coefficient (SE) }\end{array}$ & $\begin{array}{c}\text { Odds ratio } \\
(95 \% \mathrm{CI})\end{array}$ \\
\hline \multicolumn{6}{|l|}{ Patient sex } \\
\hline Female (comparator) & 6,750 & 597 & 8.8 & 0 & 1.0 \\
\hline Male & 4,858 & 506 & 10.4 & $0.156(0.067)$ & $1.169(1.028-1.329)$ \\
\hline \multicolumn{6}{|l|}{ Patient age } \\
\hline$\leqslant 50$ years (comparator) & 2,279 & 102 & 4.5 & 0 & 1.0 \\
\hline $51-70$ years & 3,243 & 321 & 9.9 & $0.637(0.124)$ & $1.891(1.494-2.395)$ \\
\hline $71-80$ years & 2,918 & 316 & 10.8 & $0.757(0.129)$ & $2.131(1.676-2.711)$ \\
\hline$>81$ years & 3,152 & 364 & 11.5 & $0.847(0.128)$ & $2.333(1.825-2.982)$ \\
\hline \multicolumn{6}{|l|}{ Consultant specialty } \\
\hline Medicine (comparator) & 5,132 & 491 & 9.6 & 0 & 1.0 \\
\hline Elderly care & 1,677 & 199 & 11.9 & $0.131(0.104)$ & $1.140(0.949-1.370)$ \\
\hline Dentistry & 16 & 2 & 12.5 & $0.612(0.453)$ & $1.843(0.411-8.271)$ \\
\hline Gynecology & 208 & 10 & 4.8 & $-0.417(0.349)$ & $0.659(0.344-1.263)$ \\
\hline Hematology & 120 & 8 & 6.7 & $-0.309(0.312)$ & $0.734(0.354-1.503)$ \\
\hline Obstetrics & 446 & 4 & 0.9 & $-1.722(0.603)$ & $0.179(0.065-0.491)$ \\
\hline Oncology & 136 & 12 & 8.8 & $-0.037(0.366)$ & $0.963(0.527-1.761)$ \\
\hline Orthopedics & 1,145 & 105 & 9.2 & $-0.021(0.142)$ & $0.979(0.784-1.223)$ \\
\hline Psychiatry & 256 & 9 & 3.5 & $-0.793(0.407)$ & $0.453(0.203-0.890)$ \\
\hline Surgery & 2,207 & 247 & 11.2 & $0.268(0.100)$ & $1.308(1.108-1.542)$ \\
\hline Urology & 255 & 16 & 6.3 & $-0.490(0.280)$ & $0.613(0.365-1.028)$ \\
\hline \multicolumn{6}{|l|}{ Calendar quarter } \\
\hline Nov-Jan (comparator) & 2,563 & 292 & 11.4 & 0 & 1.0 \\
\hline Feb-Apr & 3,362 & 338 & 10.1 & $-0.171(0.104)$ & $0.843(0.713-0.996)$ \\
\hline May-July & 2,866 & 225 & 7.9 & $-0.379(0.119)$ & $0.685(0.570-0.823)$ \\
\hline Aug-Oct & 2,817 & 248 & 8.8 & $-0.316(0.113)$ & $0.729(0.609-0.873)$ \\
\hline Constant term & & & & -2.771 & \\
\hline
\end{tabular}

NOTE. CI, confidence interval; SE, standard error.

${ }^{a}$ No. of patients with infection divided by no. of patients surveyed, expressed as a percentage.

tors that influence infection prevalence. This study found that increased age, male sex, winter season of hospitalization, and patient stay in medical and surgical wards were independently associated with increased HAI prevalence in acute care Scottish hospitals. Other surveillance studies have investigated factors that contribute to infection risk, including length of stay, presence of comorbidities, and functional status. ${ }^{8,9} \mathrm{Al}$ though these studies record multiple additional variables, they are often conducted on a small sample of institutions with potentially limited representation. To our knowledge, our survey is the first to include every acute care hospital in Scotland, with the full hospitalized patient population from 11 clinical specialties. Other surveys have failed to assess the importance of season, although this is partly because of the methodological limitations of point and period-prevalence surveys. ${ }^{8,9}$

Our second objective was to explore whether prediction models could be developed to aid infection control strategy development, at both the local and the national level. We argue that data derived from these models can be used for these purposes. There is potential for infection control teams to consider prediction estimates for specific patient groups under a given a set of conditions, namely, patient factors (age and sex), hospital specialty, and season of survey. This would enable resources and interventions to be targeted toward high-risk populations, where a high HAI prevalence is predicted (eg, elderly patients hospitalized in surgical wards during winter months). There are opportunities to channel surveillance approaches towards high-risk clinical areas or population subgroups.

Since the publication of the Study on the Efficacy of Nosocomial Infection Control, ${ }^{10}$ there has been a steady promotion of the benefits of targeted, incidence-based surveillance, compared with hospital-wide, prevalence-based surveillance. Targeted surveillance focuses preventive effort and resources on high-risk patient groups (eg, surgical patients), units (eg, intensive care units), or infection sites (eg, bloodstream infections). This surveillance approach can yield more meaningful data, because case finding is accurate and sophisticated risk adjustment is more feasible. ${ }^{4}$ However, we propose that national prevalence data can be used to inform infection control planning and highlight areas for focused incidence surveillance.

There are various limitations of our study, common to other prevalence-study designs. We could only investigate certain risk factors, because the large-scale, population-based approach and cross-sectional data collection do not allow 
assessment of the temporal relationship between exposure and outcome. It was not possible to explore system factors or detailed individual patient exposures, both of which would require detailed retrospective review or prospective methodology. We were unable to adjust for patient comorbidity within the prediction model, although there may be scope to investigate this in future research.

This study demonstrates that national infection prevalence data may be used to predict risk stratification across different patient groups and clinical settings. National HAI prevalence data should be used to target infection control interventions and to inform the national and local strategic development.

\section{ACKNOWLEDGMENTS}

We thank the national HAI prevalence project team and the NHS Scotland board, clinical staff, and infection control teams.

Financial support. This study was funded by the Scottish Executive Health Department.

Potential conflicts of interest. All authors report no conflicts of interest relevant to this article.

From Health Protection Scotland, Glasgow (J.R., S.S., G.A., S.C.), and the Infection Control Team, NHS Dumfries and Galloway (L.R.), and the Department of Public Health, University of Aberdeen, Aberdeen (J.B.), Scotland.

Address reprint requests to Dr. J. Reilly, HAI and IC Group, Health Protection Scotland, 1 Cadogan Square, Glasgow, G2 7HF (Jacqui.Reilly@hps .scot.nhs.uk)

\section{REFERENCES}

1. Strausbaugh, LJ. Emerging healthcare-associated infections in the geriatric population. Emerg Infect Dis 2001; 7:268-271.

2. General Register Office for Scotland. Scotland's Population 2006. Edinburgh, Scotland: General Register Office for Scotland; 2007:1-94.

3. European Antimicrobial Resistance Surveillance System (EARSS). EARSS Annual Report 2006. In: Team EM, ed. EARSS Annual Report. Bilthoven, the Netherlands: National Institute for Public Health and the Environment; 2006:1-162.

4. Gaynes RP, Solomon S. Improving hospital-acquired infection rates: the CDC experience. Jt Comm J Qual Improv 1996; 22:457-467.

5. Hospitals in Europe Link for Infection Control through Surveillance (HELICS). Prevalence surveys of nosocomial infections. In: Hospitals in Europe Link for Infection Control through Surveillance. HELICS; 2004:128.

6. Reilly J, Stewart S, Allardice G, et al. NHS Scotland National HAI Prevalence Survey: final report 2007. Glasgow, Scotland: Health Protection Scotland; 2007:1-239.

7. Schlesselman, J. 8.4-estimation of logistic parameters. In: Case-Control Studies: Design, Conduct, Analysis. Oxford: Oxford University Press; 1982: 244-250.

8. Gikas A, Pediaditis J, Papadakis JA, et al. Prevalence study of hospitalacquired infections in 14 Greek hospitals: planning from the local to the national surveillance level. J Hosp Infect 2002; 50:269-275.

9. Pittet D, Harbarth S, Ruef C, et al. Prevalence and risk factors for nosocomial infections in 4 university hospitals in Switzerland. Infect Control Hosp Epidemiol 1999; 20:37-42.

10. Haley RW, Hooton TM, Culver DH, et al. Nosocomial infections in U.S. hospitals, 1975-1976. Am J Med 1981; 70:947-959. 